\title{
A Plant-and-Play Wireless Sensor Network System for Gate Monitoring
}

\author{
Raghuram S Sudhaakar, Ameya M Sanzgiri, Murat Demirbas, Chunming Qiao \\ Computer Science and Engineering, University at Buffalo, Buffalo, NY
}

\begin{abstract}
In this work, we present a practical plant-and-play wireless sensor network system for entry-exit monitoring. Our system is easily configurable and robust, making it feasible to be deployed in a wide range of entry-exit monitoring applications. At the core of our system lies a novel MAC protocol that is self-synchronizing. Notably, our MAC protocol allows the nodes to maintain a very low duty cycle, while also enabling quick synchronization of the nodes (when needed) for a consistent classification of entry or exit events. We have deployed this system for monitoring a faculty parking lot at our university and integrated it with an SMS notification system to provide information on the availability of parking spots on demand. We also present the parking lot occupancy trends obtained through this deployment, and discuss some of the reliability issues encountered.
\end{abstract}

\section{INTRODUCTION}

Wireless sensor networks (WSNs) have been adopted by scientists and specialists for several environment/habitat monitoring and military surveillance applications [1]-[5]. We believe that, in order for the WSNs to receive a wider community adoption, and as a result, start riding the benefits of mass production and the hidden hand of the market, we need to focus our attention more towards the urban applications of WSNs.

In this work, we consider entry-exit monitoring applications that are useful for many urban monitoring applications, such as, counting the number of goods moved through loading docks, mining the behavior of customers shopping in the malls, and monitoring the occupancy in public buildings to devise more efficient evacuation strategies. In this paper, we focus on monitoring parking lot utilization as our case study.

Parking lot monitoring application - A survey of the parking facilities at our university shows that there are 36 fully functional parking lots distributed over two square miles of campus with a total capacity of 9000 cars. However, there are about 30,000 faculty, staff and students on campus, and at peak hours there is an observable congestion in parking lots. Commuters spend a considerable amount of time as well as fuel searching for empty parking spots. Significant savings are possible by deploying a practical WSN-based system to monitor the occupancy of the parking lots and providing this information to the commuters on-demand via Short Message Service (SMS).

Although the problem of gate monitoring has been studied before (see Section II for previous work), the solutions provided require substantial infrastructure and would not be viable for most existing parking lots. The novelty of our system is that it incorporates a Plant-and-Play approach that eliminates the need for costly infrastructure, making it suitable for deployment in existing as well as new parking lots.

Several challenges exist for developing such a practical Plant-and-Play gate monitoring system. The main challenge is designing a system that is inexpensive to deploy, easy to operate, and hassle-free to maintain. The system should also be flexible and parameterizable for the requirements of various applications (such as entry-exit detection of people, bicycles, or trucks) without compromising robustness and fault tolerance.

\section{A. Our Contributions}

We address these challenges by designing, implementing, and deploying a plant-and-play WSN-based gate monitoring system. More specifically, we list our contributions as follows:

1. A parametrized/configurable gate monitoring system: We designed our system to be easily configurable, to match the requirements of the application, by adjusting a set of key parameters we identified. We present an extensive analysis of these key parameters and discuss the effects of these parameters on the accuracy and the performance of our system.

2. A self-synchronizing MAC protocol for energy efficient monitoring: We designed and implemented a novel MAC protocol for our entry-exit monitoring system that enables each node to independently synchronize itself to the other nodes in singlehop with negligible overhead. Our MAC protocol maintains a very low duty-cycle by keeping the node radios in sleep mode $100 \%$ of the time in the absence of detections. Yet, when triggered by a detection our MAC protocol synchronizes the node radios quickly for a consistent classification of entry or exit events. This low duty-cycle operation gives the nodes in our system an estimated lifetime of 6 months while running on two AA batteries.

3. A decentralized algorithm for entry-exit detection: We designed and implemented a simple decentralized algorithm to compute the direction of vehicle movement independently at each node in the network. In order to accurately classify the events as entry or exit, the algorithm uses the detection information received from the other nodes as well as the local detection information as input. 
4. Results from a real world deployment: We deployed our system for monitoring of a faculty parking lot with 280 car capacity, and integrated it with an SMS notification system to provide the users the parking availability information on demand. We present the parking lot occupancy trends obtained through this deployment, and discuss some of the reliability issues encountered.

\section{B. Outline}

The rest of the paper is organized as follows. After reviewing the related work in Section II, we present our system design in Section III, including our design of a self-synchronizing MAC protocol, motion detection mechanism and decentralized entry-exit classification algorithm. We present an analysis of the design parameters in Section IV and an analysis of the energy consumption in Section V. Finally, in Section VI, we present results from our faculty parking lot deployment and discuss reliability issues.

\section{RELATED WORK}

Most prevalent fee-based parking lots use gates at all entry and exit points of the parking lot. The driver of the car is issued a tag at the entry point and the system registers the entry of the car. When the driver presents the tag again at the exit point, the system registers the exit of the car. This way, the overall occupancy of the parking lot is known at all times. In [6], the authors describe a system that uses RFID-based tags to monitor the movement of cars.

Some modern parking lots use devices like pressure sensors at each parking spot to detect the presence of a car. Further advances in technology [7] also enable the use of sensors that detect the change in the magnetic field caused by the presence or movement of a car to detect whether a parking space is occupied. By collecting this information from each of these sensors at a central control unit, the availability of empty parking spots can be determined at all times. Such systems offer the ability to provide drivers with information on the exact location of empty parking spaces in the parking lot.

However, these methods require considerable investment for setting up the required infrastructure and also involve significant operational and maintenance costs, making their deployment in existing parking lots mostly infeasible.

In [8] [9] [10] [11], advanced image processing techniques are described that are capable of detecting the occupancy of a parking lot.

These techniques work well only under ambient light conditions and require complete aerial coverage of the parking lot. Under poor lighting condition, such as at night, the efficacy of these system may significantly reduce. Further, it might not be possible to obtain an aerial image of the complete parking lot for underground or basement parking lots.

In [12] the authors make use of WSNs to detect cars and provide information to a central node at a police booth which in turn communicates with a central server using optical communication techniques. However, this solution also requires significant infrastructure.

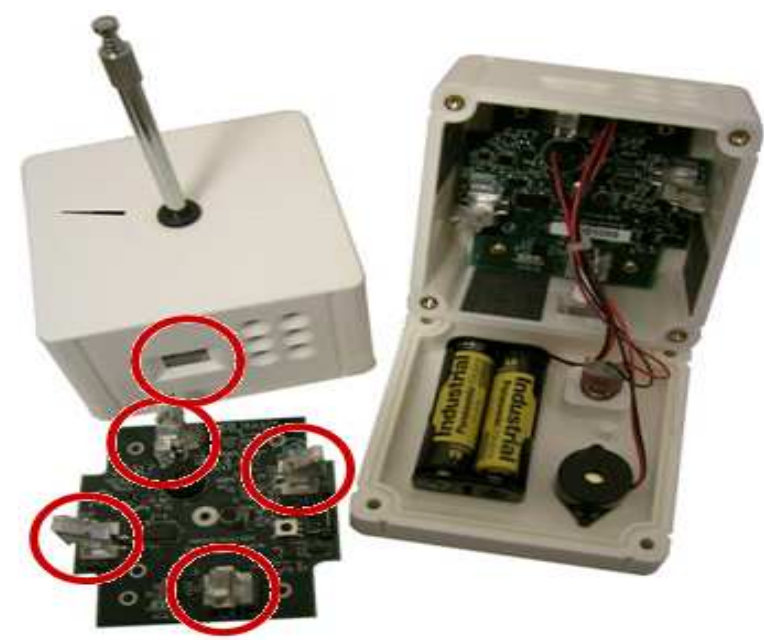

Fig. 1. XSM circuit board with integrated sensors and the XSM ruggedized enclosure. The red circles show the PIR sensors on the board as well as on the enclosure (Figure courtesy EECS, UC Berkeley and CrossBow Technology)

In contrast, a major contribution of our work is the design and development of low-cost and robust system that works efficiently under various physical and environmental conditions and has minimal infrastructure requirements.

In [13] the authors present a self-stabilizing MAC protocol using a TDMA-based approach that relies on global timesynchronization at all the nodes.

In [14] the authors lift the global time synchronization requirement, and describe a self-stabilizing distributed MAC protocol using the concept of frames. However, practical issues crucial for a deployment such as energy consumption and latency have not been addressed in the protocol.

In contrast, our MAC protocol addresses the issues of energy consumption and communication latency while also being self-stabilizing and distributed without the need for a global time synchronization.

\section{System Design}

Overview. We use Extreme Scale Motes $(X S M)$ from CrossBow Technologies [15], which are based on the Mica2 platform. The XSM motes are specifically designed for rugged use and are made as a complete enclosed unit sturdy enough for deployment under various conditions. The $X S M \mathrm{~s}$ are equipped with a telescopic antenna that provides a wide radio range (in comparison to other motes) of about 50 meters. These features of the $X S M$ make it a suitable choice in our work. The XSMs are also one of the few available motes that have built in Passive Infra-Red (PIR) sensors which can perform robust motion detection. The PIR sensors have a high tolerance towards ambient conditions and can operate efficiently under varying lighting conditions. Figure 1 shows the circuit of the $X S M$ 's.

Figure 2 shows our system setup and placement of the XSM nodes for entry-exit monitoring. We use a decentralized algorithm that efficiently classifies the readings generated by the PIR sensor to detect and differentiate movement of various objects. 


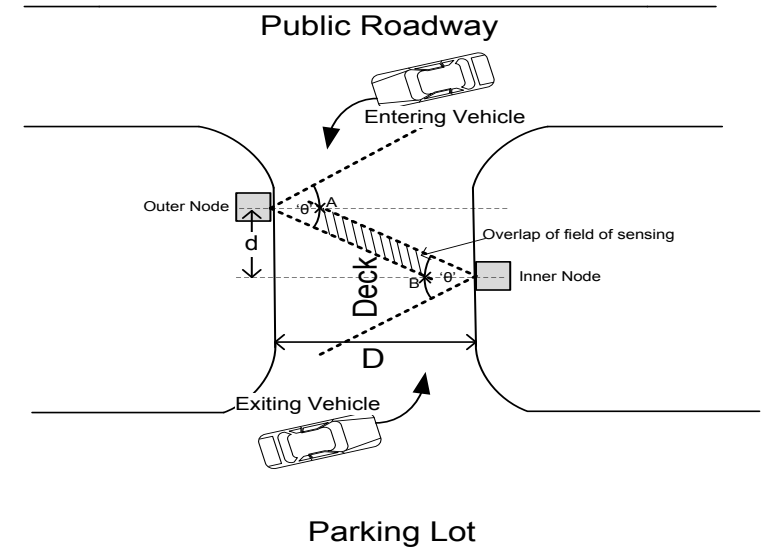

Fig. 2. Placement of XSM nodes for entry-exit monitoring

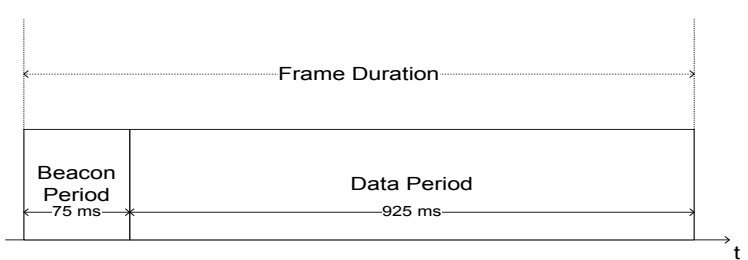

Fig. 3. MAC frame structure

In order to compute the direction of movement in a decentralized manner, it is necessary that the nodes in the network are synchronized. For this purpose we use a self-synchronizing MAC protocol that enables the nodes to independently synchronize themselves with other nodes with minimal overhead. The MAC protocol also offers mechanisms for energy efficient communication which is needed for exchange of information between the nodes.

On receiving the information from the other nodes, each node then executes the decentralized algorithm, with the local detections and the data received from the other nodes as input, to determine the direction of movement of the vehicle. By storing and processing these entry-exit events, the current occupancy level of the parking lot is easily calculated.

Sections III-A, III-B, III-C describe the self-synchronizing MAC Protocol, motion detection using the PIR sensors, and the decentralized algorithm, respectively.

\section{A. Self-Synchronizing MAC Protocol}

In this section we describe a simple yet effective MAC protocol that allows each node to synchronize with all the other nodes in singlehop independently and with minimal overhead. The MAC protocol has a simple frame structure as shown in Figure 3. The total frame duration is one second. The first $75 \mathrm{msec}$ from the start of the frame is allocated for beacons and is called the Beacon Period (BP). The rest of the $925 \mathrm{msec}$ is allocated for data transmissions and is called the Data Period $(D P)$.

When the node is switched $\mathrm{ON}$ it scans the channel for existing beacons. If it does not find any existing beacon, then the node installs its beacon at a randomly chosen time and this will be the start of the frame for the node and all the nodes that come up subsequently. Otherwise, if the node finds beacons already being transmitted on the channel, it adopts the start of the first beacon on the channel as the start of the frame and installs its beacon at an available empty slot within the beacon period.

In this approach there is a possibility that there might be beacon collisions if a few nodes are switched $\mathrm{ON}$ at the same time. Hence we introduce a startup delay which is randomized based on the node ID. Further, the protocol also includes mechanisms to detect beacon collisions that are based on randomly listening in the slot it is supposed to transmit its beacon. A combination of these two techniques ensures that beacon collisions are completely avoided.

There is no information exchange during this phase and each node independently decides where to install its beacon. Since all the nodes agree on the start of the frame, which is the start of the first beacon, synchronization can be achieved among all the nodes in singlehop. This procedure is executed only by the new nodes at the time of joining the network without information exchange with existing nodes. Thus the overhead of time synchronization is minimal and protocol scales very well.

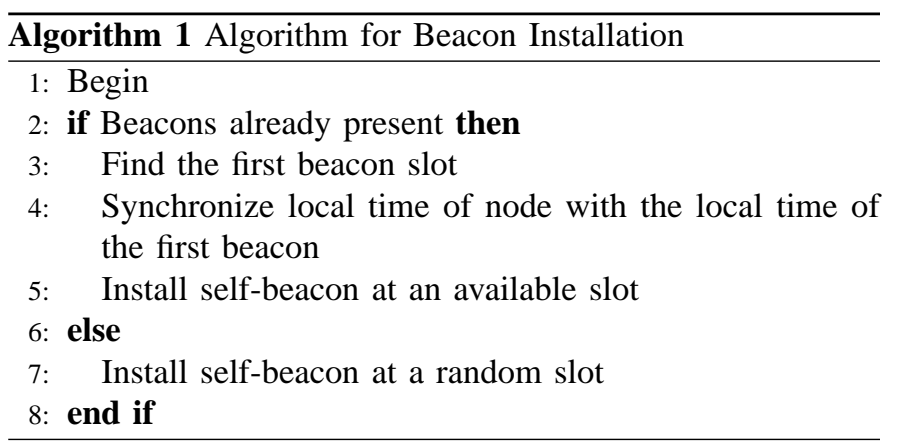

Whenever data is generated by the sensors it is passed on to the MAC module, which buffers the data until the next BP. While transmitting the beacon during the BP, the node includes information on when it will transmit the data during the DP. The node radios are awake during the entire beacon period so that they can listen to the beacons transmitted by other nodes. By listening to the beacons of the other nodes each node can independently determine when it should wake up during the data period to receive information from other nodes. Consequently, there is a significant power saving, as the radio is awake precisely when it is required to receive data while sleeping at all other times.

Implementation details. In order to enable the nodes to have the same absolute value of the local system time, each node also transmits its local system time in its beacon. Hence the nodes that come up after the first node can synchronize the absolute values of their system times to the first node. This scheme provides an easy method for all the nodes to have approximately the same system time in a distributed manner without external supervision. It should be noted that the system times of all the nodes will not be exactly the same due to propagation delays and local clock drifts, however, we have experimentally determined in our deployment that it is possible to achieve a synchronization with an error of less than $5 \mathrm{msec}$ 
between any two nodes using the $\mathrm{CC} 1000$ radio module. (Finer synchronization can be easily achieved by using higher data rates and better radio modules.) Thus, in our application, the timestamp in the message of a node can be directly used by the other node without any correction as they are synchronized to within $5 \mathrm{msec}$. This is very useful as it reduces processing overhead for each packet, in terms of offset correction, and hence aids in reducing the overall complexity.

The packet size for the beacon is 14 bytes and as a result the transmission duration of a beacon (inclusive of preamble, guard and other standard bits), assuming the standard CC1000 transmission rate of $19.2 \mathrm{kbps}$, is $7.5 \mathrm{msec}$. Consequently, only 10 nodes can be supported by this MAC protocol at any given instant of time. This constraint can be easily overcome by adjusting the beacon period and data period, depending on the number of nodes that require to be supported by the system. However, we expect that it will not be necessary to use more than 4-5 nodes in any parking lot gate.

\section{B. Motion Detection Using PIR Sensors}

We use Passive Infra-Red(PIR) sensors to detect movement. The PIR sensors are very popular due to their low power consumption, low cost and broad availability. They are the central components in many motion sensors for automatic lighting, security systems, and electric doors. Among the many available motion detection sensors, PIRs are the most tolerant to varying environmental situations and can operate efficiently under different lighting and weather conditions. Further, they are considerably more sensitive, have a significantly larger field of sensing and are less prone to false detections as compared to other motion detection sensors, such as magnetometers or acoustics based sensors. In [16], it is suggested that a combination of PIRs, magnetometers, and acoustic sensors can be used to effectively detect and classify the movement of various objects. However, under practical deployments scenarios, the field of detection and the rate of false alarms of the magnetometers and the acoustic sensors are very high. Thus, we choose to use only PIRs in our parking lot occupancy monitoring application.

The Kube Electronics C172 pyroelectric sensor [17] [18] is at the core of the PIR subsystem. Each PIR sensor in our XSM mote has a 110 degree field-of-view and is capable of sensing motion at up to a distance of $25 \mathrm{ft}$. The XSMs have four PIR sensors, mounted at 90 degree intervals, so that their fields-of-sensing overlap slightly. However, in our application we need to detect movement of vehicles at the entrance to the parking lot and this can be done by using only one of the PIRs that is facing the roadway. Accordingly, the other three PIR sensors on the $X S M$ are turned off at all times.

Figure 4 shows the graphical representation of the readings generated by the PIR sensors for movement of different objects. The waveform shows that the PIR sensor readings maintain a steady value of about $0 \times 1 F F$ when no movement is detected and generate an approximately sinusoidal waveform when movement is detected. Consequently, the most efficient

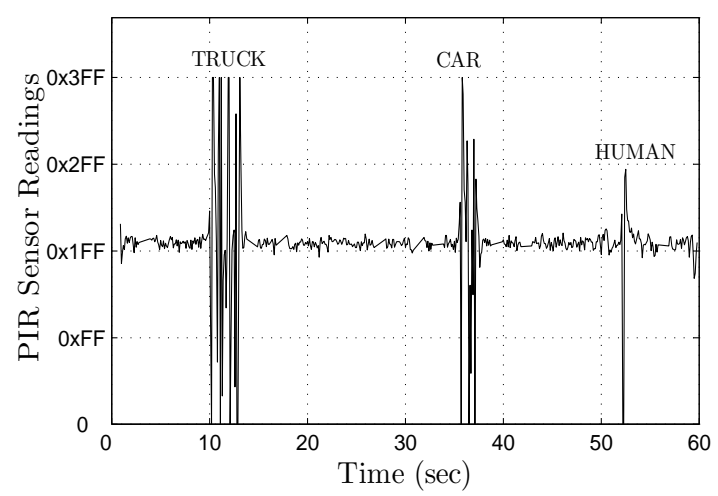

Fig. 4. PIR Waveform for the detection of movement of various objects

way to determine if movement is occurring is to continuously monitor the gradient of the waveform.

In our implementation, we activate the PIR sensor once every $T$ milliseconds and sample the readings generated by the sensor. More specifically, if the gradient is greater than a particular threshold, we decide that some movement has been detected and in order to obtain a finer resolution on the time scale, we increase the sampling frequency of the PIR sensors to once every $t$ milliseconds $(t \leq T)$. We continue to read the PIR sensors at a frequency of once every $t$ milliseconds until the gradient falls below a small value, indicating that no movement is being detected. Then, we revert to sampling every $T$ milliseconds to conserve energy. The start and end times of the detections are recorded to be supplied as one of the inputs to the decentralized entry-exit detection algorithm.

Algorithm 2 formalizes these steps. Although the algorithm is very simple, the results from our field tests show that it is effectively able to detect different types of vehicles, ranging from small cars to buses, at a significantly wide range of speeds.

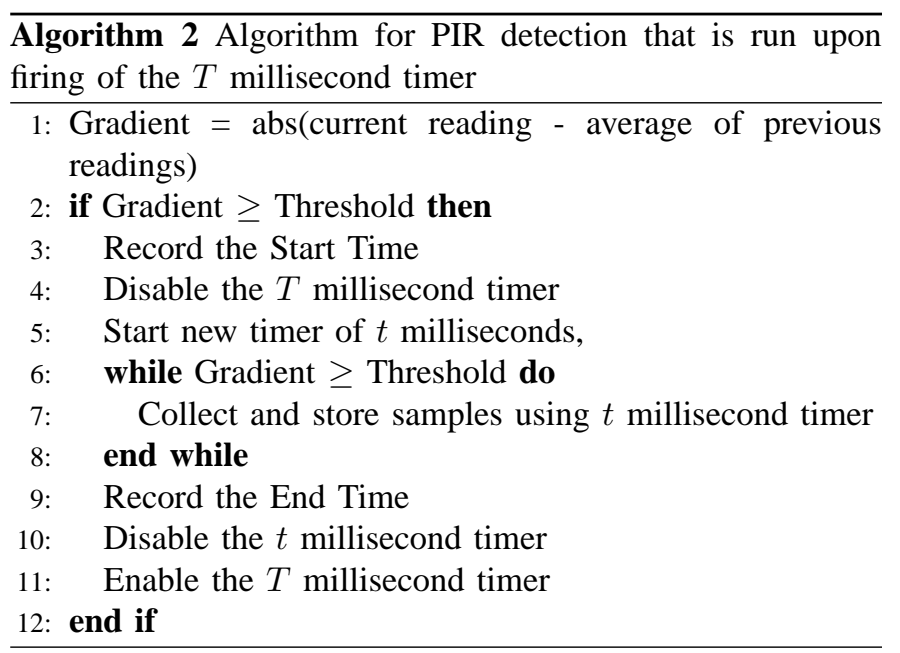

It is important to note that the values of $T$ and $t$ are important design parameters for the system as the successful detection of movement at varying speeds is directly affected by their values. For example, if $T$ is too large then a fast moving vehicle might not be detected at all and if $T$ is too small then a vehicle that is moving slowly through the field 


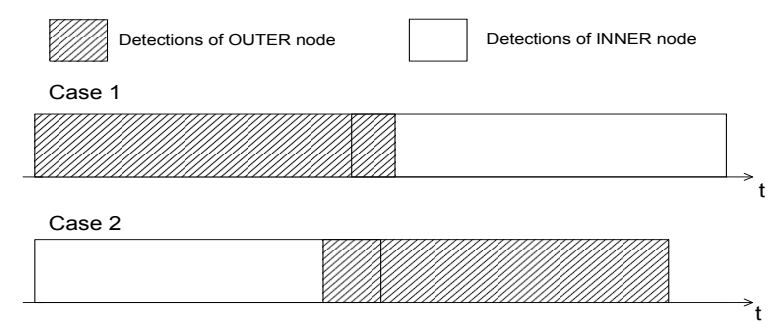

Fig. 5. Timing of the readings for various cases

might trigger a false detection. In Section IV we develop the mathematical analysis for the selection of these parameters.

\section{Decentralized Entry-Exit Detection Algorithm}

In this section we present our simple decentralized algorithm that is executed by each node for entry-exit detection. The algorithm takes the start and end times of the PIR detections by the node as the local input and the start and end times received from the other node as the received input. Using this information the algorithm accurately determines the direction of movement of the vehicle through the fields of the nodes, and consequently, decides whether a vehicle entered or exited the parking lot.

As shown in Figure 2, the nodes are designated as Outer and Inner nodes depending on their proximity to the parking lot. The node that is closer to the parking lot is designated the Inner node while the other node is designated the Outer node.

The basic idea behind the algorithm can be explained easily by a visualization of the start and end times of the detections of both the nodes on a time scale as shown in Figure 5. If the Outer node started detecting movement before the Inner node and stopped detecting before the Inner node then then the algorithm decides that the direction of movement of the vehicle was in the direction from the Outer to the Inner node, and hence that the vehicle entered the parking lot. Using a dual argument, if the Inner node started and ended detections before the Outer node then the algorithm decides that the vehicle exited the parking lot. Note that the situation described here occurs only when the fields of the two sensor overlap in an optimal manner as we discuss in Section IV-A.

\section{Analysis of Design Parameters}

In this section we discuss the various parameters that govern the performance of the system and develop mathematical formulae for their design. We also discuss the effects of these parameters on the accuracy of the decentralized algorithm and provide the reader an insight on how to determine the best possible configuration for a given application scenario by way of presenting suitable design parameters for some of these scenarios.

\section{A. Placement Of Nodes}

In order to achieve a generic system design, we allow all the entry/exit points to the parking lot to be bidirectional and allow traffic in both directions. We also allow the cars can travel at a significantly wide range of speeds through these

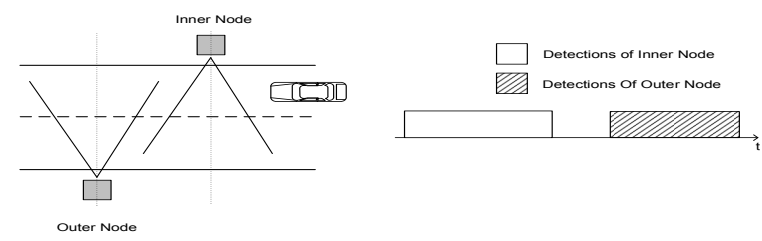

(a) Distance of Separation too large

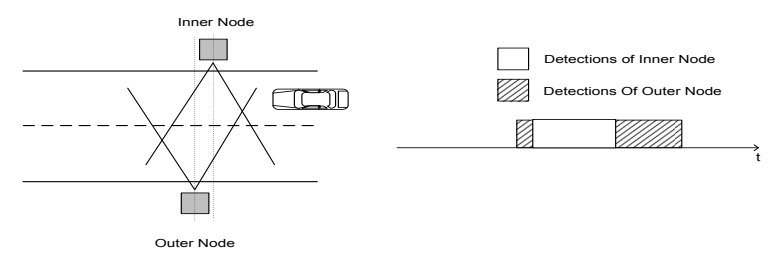

(b) Distance of Separation too small
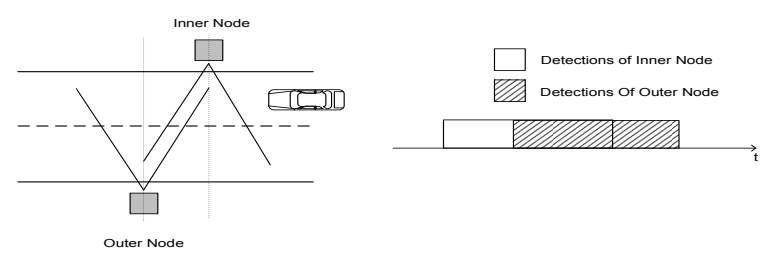

(c) Optimal Distance of Separation

Fig. 6. Effect of the Distance of Separation of the nodes on the detections

entry/exit points. The nodes are placed on the opposite sides of the entrance as shown in Figure 2.

An analysis of the decentralized algorithm presented in Section III-C shows that the accuracy is considerably improved if the start and end times of the detections of the two nodes are separated as shown in Figure 5. More specifically, the vehicle should enter the field of the sensor that is nearer to its lane of travel and then enter the field of the sensor that is farther from its lane of travel. It is also necessary that it leaves the field of sensing of the node that is nearer to its lane of travel before it leaves the field of sensing of the sensor that is farther.

In order to ensure that the detection of the nodes are optimally separated, we require that the nodes and hence their fields of detections are separated by an optimal perpendicular distance. We define this parameter as the Distance of Separation (' $d$ ') as in Figure 2. The distance of separation governs the region of overlap of the fields of detections of the two nodes. We require that the fields of detections are neither completely disjoint as shown in Figure 6(a) nor should they be completely overlapping as shown in Figure 6(b). The optimal sequence of detections occur when the nodes are separated by an optimal ' $d$ ' as shown in Figure 6(c).

Since the distance of separation is critical to the accurate operation of the decentralized algorithm, we give a procedure to calculate the distance of separation as follows.

We have experimentally determined that the accuracy of the system was highest, when the overlapping edge of the field of sensing of each node passes through the intersection of the perpendicular drawn from the other node and the line of travel of the vehicles on the farther lane. Without loss of generality, we approximate the line of travel of the vehicles to be along the middle of each lane. By exploiting the geometry, we then 
TABLE I

Distance Of Separation For Various Angles and Deck-WidThS

\begin{tabular}{ccc}
\hline $\begin{array}{c}\text { Deck Width } \\
(\mathrm{m})\end{array}$ & $\begin{array}{c}\text { Angle of Sensing } \\
\text { (degrees) }\end{array}$ & $\begin{array}{c}\text { Distance of Separation } \\
(\mathrm{m})\end{array}$ \\
\hline \multirow{3}{*}{4} & 110 & 4.284 \\
& 90 & 3 \\
70 & 2.1 \\
\hline 8 & 110 & 8.568 \\
& 90 & 6.0 \\
& 70 & 4.2 \\
\hline \multirow{3}{*}{10} & 110 & 10.71 \\
& 90 & 7.5 \\
& 70 & 5.25
\end{tabular}

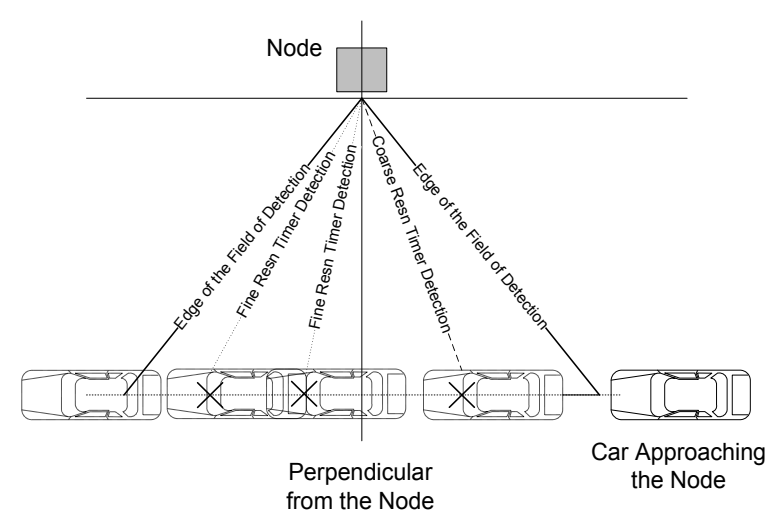

Fig. 7. Ideal scenario for detecting movement of a car through the field of the sensor

write the expression for the optimal distance of separation as follows

$$
d=\tan \left(\frac{\theta}{2}\right) * 0.75 * D \text { meters }
$$

Table I shows the optimal values of $d$ for different Deck Widths $(D)$ and Angles of Sensing $(\theta)$.

\section{B. Timer Durations for the PIR Sensors}

We need to consider that the speeds of the vehicles passing through entry/exit of different parking lots may vary. Since the PIR readings are sampled every $T$ seconds (according to the coarse resolution timer), the speed of the vehicles directly affect the number of samples obtained. A low rate of sampling will lead to false positives/negatives with respect to motion detection while a high rate of sampling will lead to unnecessary depletion of energy.

From Figure 2 it is obvious that the node that is closer to the lane of travel of the vehicle should always start sensing first, while the node that is farther away should start sensing later. Figure 7 shows a car traveling from right to left. In the worst case scenario it is possible that the PIR sensor was sampled just before the vehicle entered the field of sensing. For accurate detection of movement using the algorithm described in Section III-B, we require that at least one detection of the vehicle passing through the field of sensing is made before the vehicle crosses the perpendicular drawn from the node. This is an arbitrary distance selected by us to exploit the geometry. The rationale behind choosing this distance is to allow for
TABLE II

PARAMETERS FOR THE PARKING LOT MONITORING APPLICATION

\begin{tabular}{llcccc}
\hline $\begin{array}{l}\text { Speed } \\
\text { Range } \\
(\mathrm{mph})\end{array}$ & $\begin{array}{l}\text { Avg. } \\
\text { Speed } \\
(\mathrm{mph})\end{array}$ & $T_{\max }(\mathrm{sec})$ & $T_{\text {avg }}$ & $t_{\max }$ & $t_{\text {avg }}$ \\
\hline $5-25$ & 15 & 0.127 & 0.213 & 0.0426 & 0.0709 \\
$26-40$ & 33 & 0.0795 & 0.0967 & 0.0267 & 0.03226 \\
$41-60$ & 50.5 & 0.0532 & 0.06325 & 0.01774 & 0.021085 \\
\hline
\end{tabular}

the fine resolution timer to be started and to obtain at least a few readings. We have experimentally corroborated that this method improves the accuracy greatly.

Ideally $T$ should be such that the maximum distance the vehicle can cover within the duration $T$ is less than half the length of the field of sensing along the line of travel of the car. It should also be noted that the length of the field of sensing along the line of travel of the vehicle is lesser for the node that is nearer to the lane of travel of the vehicle as compared to the node that is farther away. Hence we consider the smaller of the two distances, which is given by

$$
\tan \left(\frac{\theta}{2}\right) * \frac{D}{4} \text { meters }
$$

Thus, if the speed of the car were to be $v$ meters per second and the time taken for the car to travel from the edge of the field of sensing to the perpendicular drawn from the sensor is equivalent to the resolution of the timer, the value of $T$ is given by

$$
T=\left(\tan \left(\frac{\theta}{2}\right) * 0.25 * D\right) * \frac{1}{v} \sec
$$

Recall that our system also provides a fine resolution timer whose significance is to enhance the reliability readings and improve the accuracy of the system. As per the rationale of the initial timer mentioned above and the need for at least $n(n \geq 2)$ detections, before the car exits from the field of sensing of a sensor, the resolution of the fine timer is calculated as

$$
t=\tan \left(\frac{\theta}{2}\right) * 0.25 * D * \frac{1}{n v} \sec
$$

The concept of the detection using two timers is also depicted in Figure 7. Furthermore, Table II gives the resolution of the timers suitable for various speed ranges.

\section{ANALYSis OF ENERGY CONSUMPTION}

In this section we present an analysis for the total energy consumption of the nodes. The energy consumption of the nodes depend on two operational modes: 1 . when there are detections and the nodes are in the ACTIVE mode, and 2. when there are no detections and the nodes are in the IDLE mode. The total energy consumption for each state can be further categorized into the energy consumption of Radio, PIR Sensors and Processor modules. In the following, we consider the ACTIVE and IDLE modes of these modules and their corresponding energy consumption in those states to derive an approximate energy consumption model. 
1) Idle Mode: The energy consumed by the Radio module can be categorized as energy consumed when transmitting, receiving, and when the radio is OFF. From the brief analysis in Section III-A we infer that, in a time frame of one second, the radio module transmits the beacon for $7.5 \mathrm{mSec}$, receives a beacon for $7.5 \mathrm{mSec}$ and is OFF for the remaining $985 \mathrm{mSec}$. The power consumption in these states are $48 \mathrm{~mW}, 24 \mathrm{~mW}$ and $3 \mu W$, respectively, resulting in a total energy consumption of $542.949 \mu J$.

Similarly the Processor module is in the ON state for $20 \mathrm{mSec}$ and in the SLEEP state for the remaining $980 \mathrm{mSec}$ and the power consumption in these states are $30 \mu \mathrm{W}$ and $24 m W$ respectively. The total energy consumption by the Processor module is $509.4 \mu \mathrm{J}$. The PIR component of the nodes is in the $\mathrm{ON}$ state for the entire duration, and at a rate of $0.88 \mathrm{~mW}$ consumes $0.88 \mathrm{~mJ}$ of energy.

Thus the total energy consumed in this mode is $1.932 \mathrm{~mJ}$.

2) Active Mode: The detection of movement results in the exchange of messages between the nodes and this increases the duration for which the Radio and Processor modules are active. A closer analysis shows that the radio module transmits for $22.5 \mathrm{mSec}$, receives for $22.5 \mathrm{mSec}$ and is OFF for the remaining $955 \mathrm{mSec}$. Similarly, the Processor module is ON for $80 \mathrm{mSec}$ and is OFF for $920 \mathrm{mSec}$. The PIR module continues to remain on for the entire duration. Proceeding with the energy calculations as above we can show that the total energy consumed in this mode is $4.45 \mathrm{~mJ}$.

On average, the number of detections during a period of 24 hours was estimated to be 1500 on weekdays and 200 on weekends, which gives the average number of detections per day to be 1128. From the above analysis we calculate the total energy consumption per day as approximately $170 \mathrm{~J}$. Since the average energy provided by $2 \mathrm{AA}$ batteries is about $30 K J$, this translates into an average lifetime of approximately 6 months for each node. Hence, the running and maintenance costs of our system are relatively low.

\section{PARking Monitoring ApPlication}

\section{A. Deployment Details}

We deployed our system at a faculty parking lot with 280 car capacity, where the speed limit range was ' $15-20$ ' miles per hour. The parking lot had a single entry/exit point with the width of entrance road measured to be 2meters. The two monitoring motes were mounted on PVC pipes of length ' 0.5 ' meters to alleviate the ground-effect (reflections of radio waves from ground). Figure 8 shows an aerial picture of our deployment setup.

The basestation was deployed at a nearby building inside an office, that resides at a height of 15 meters and at a distance of 60 meters away from the nearest node. The basestation consisted of a laptop and an XSM mote connected to the laptop to act as a gateway between the laptop and the monitoring XSM motes deployed at the parking lot. The basestation laptop had access to the Internet, so we could remotely access the basestation to get the logged data as well as issue commands

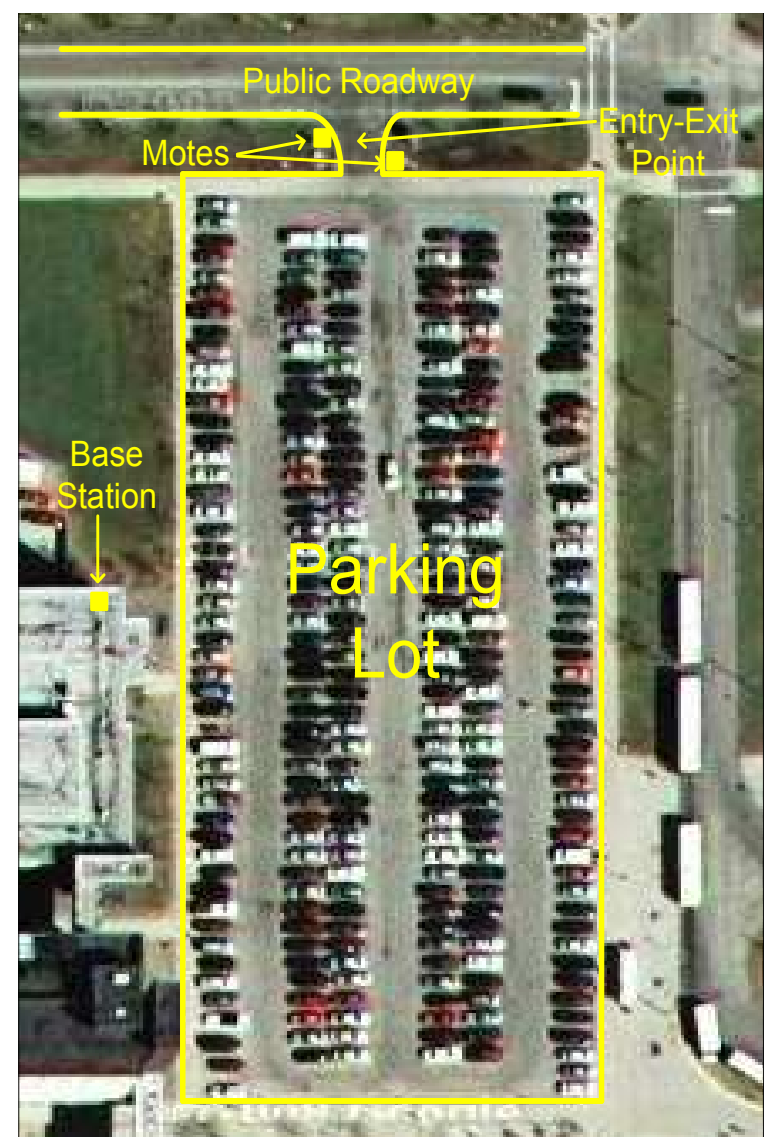

Fig. 8. Aerial view of our parking lot deployment

to the basestation to change the parameters of the deployed nodes on the fly.

In order to make the occupancy of the parking lot information useful to the commuters, this has to be disseminated to them in real time based on demand. To this end, we deployed a rudimentary notification system by employing the Short Message Service (SMS). The commuter can obtain parking lot availability data on demand by sending an email (via SMS) to the notification facility. At the basestation, we run a script that parses the received emails, and sends a reply which results in the email being sent as an SMS over the internet. Alternatively commuters can subscribe to the notification facility by providing their mobile numbers to receive based on their indicated estimated arrival times.

\section{B. Parameters Chosen}

The parameters for our deployment were set based on the results presented in Tables I and II. The distance of separation of the motes was calculated as $d=3.5 \mathrm{~m}$. The coarse resolution timer was calibrated to fire every 500 milliseconds and the fine resolution timer was calibrated to fire once every 100 milliseconds. To avoid frequent false detections and also compensate for slowing down of the cars while entering the parking lot, a decay parameter was set to stop the fine resolution timer. The configuration of all these parameters was done remotely using the JAVA GUI application we deployed at the basestation. 


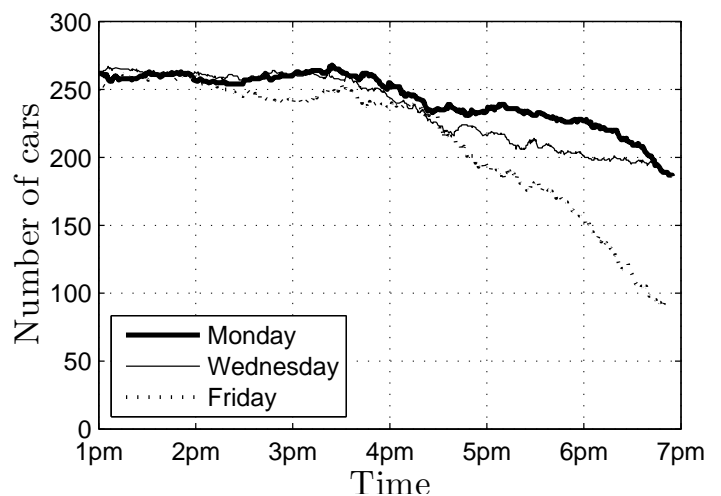

Fig. 9. Occupancy of the parking lot on weekdays (1pm to $7 \mathrm{pm}$ )

\section{Results from the Deployment}

In order to verify the performance of the system and also obtain useful statistics on the occupancy of the parking lot we deployed our system over a span of couple of weeks in September and October 2008. We verified the accuracy of the system by occasionally taking manual counts of the occupancy of the parking lot during the deployment.

Figure 9 shows a comparison of the occupancy on three typical weekdays between $1 \mathrm{pm}$ and $7 \mathrm{pm}$. We can see that the occupancy trends are very similar for all the days between $1 \mathrm{pm}$ and $4 \mathrm{pm}$. After $4 \mathrm{pm}$ there is a notable decrease in the occupancy on Friday as compared to Monday and Wednesday. We can also see a consistent pattern in the slight increase in the occupancy between $3 \mathrm{pm}$ and $4 \mathrm{pm}$ as the faculty parking lot is open to students after $3 \mathrm{pm}$ on weekdays.

Fig.10 shows the occupancy trends on a weekend. It can be seen that before $12.00 \mathrm{pm}$ the occupancy on Sunday is higher than that on Saturday. This was due to a prayer service congregation that is held on campus every Sunday. After 12.00 $\mathrm{pm}$, however, the occupancy on a Sunday is lower than on a Saturday.

Figure 11 shows the occupancy from $6.30 \mathrm{pm}$ on a weekday night to $10.30 \mathrm{am}$ the next morning. We can see that more than 100 cars remained parked in the parking lot during that particular night. Further, there is a definite pattern in the arrival of people to the campus as can be seen from the occupancy between 6 am and $10 \mathrm{am}$. There is a spurt of arrivals between $6 \mathrm{am}$ and $7 \mathrm{am}$ and then again from $8.30 \mathrm{am}$ to $10.30 \mathrm{am}$. From 7 am to 8.30 am there is very little activity.

Figure 12 shows the occupancy trends in the morning of the U.S. Presidential Election Day (11/04/08) and another typical Tuesday $(10 / 28 / 08)$. There is very little difference in the occupancy pattern.

Figure 13 presents the trend on a night when a football game was being played at the university stadium. The game was scheduled to start at $7.30 \mathrm{pm}$ that day and correspondingly, we can see an increase in the occupancy up to $7.45 \mathrm{pm}$ as the lot was open to public and students after $3 \mathrm{pm}$ that day.

Our deployment results suggest that there are definite and significant patterns in the parking lot occupancy. Although we have been able to obtain a relatively small data set from our deployments (due to administrative difficulties), in future, we

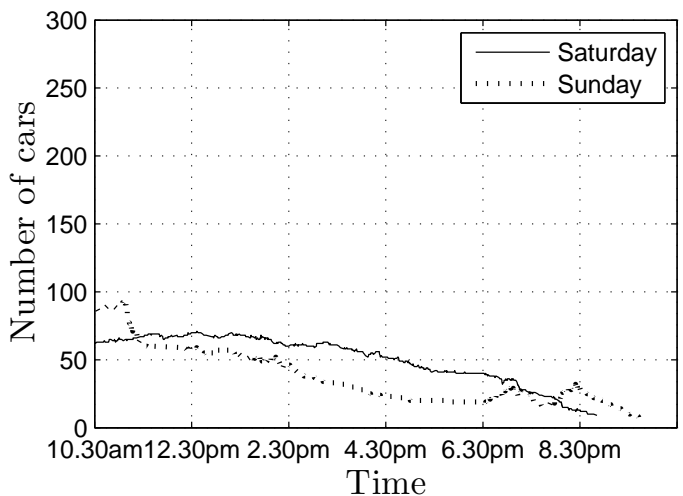

Fig. 10. Occupancy of the parking lot on weekends (10.30am to $8.30 \mathrm{pm})$

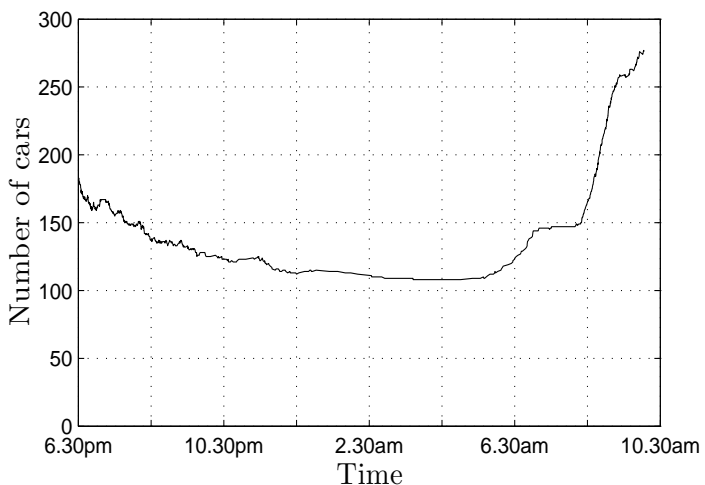

Fig. 11. Overnight occupancy of the parking lot on a weekday $(6.30 \mathrm{pm}$ to 10.30am)

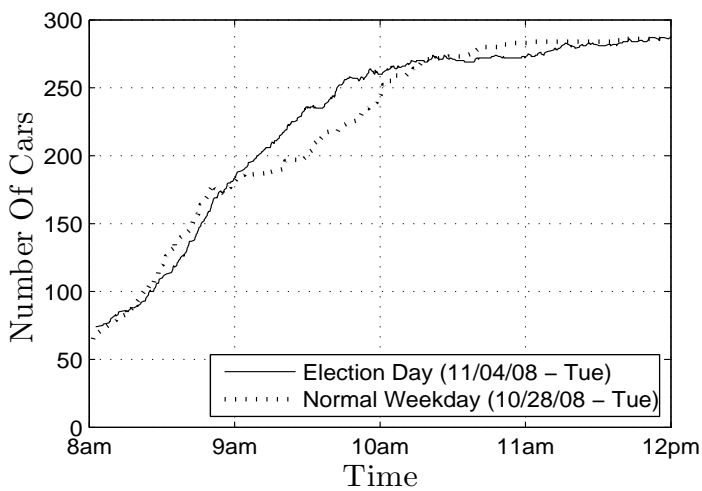

Fig. 12. Occupancy of the parking lot on the Election Day (8am to 12pm)

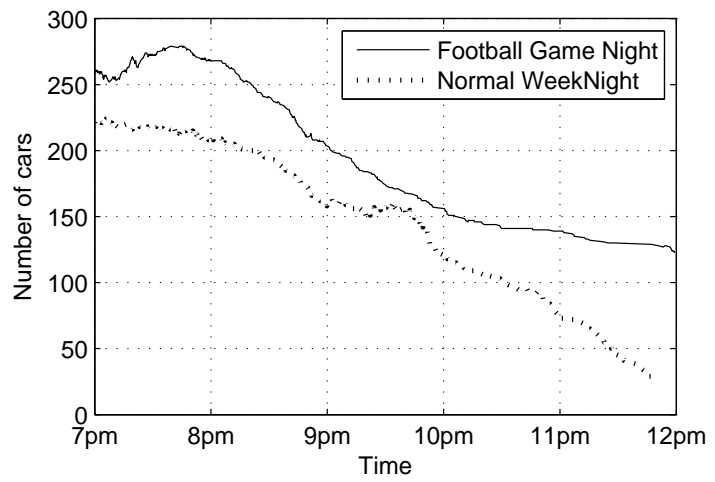

Fig. 13. Occupancy of the parking lot on a Football Game Night (7pm to 12am) 
plan to deploy this system on a much larger scale to collect a sufficiently large data set to mine patterns.

\section{Reliability issues}

In terms of network connectivity the system performed very well. An analysis of the packet error rates shows that it was less than $10^{-5}$. We attribute this to the fact that the XSM radio module operates in the $900 \mathrm{Mhz}$ band that is largely tolerant to various ambient conditions, and that the telescopic antennas of the XSMs improve their reliability of communication.

The PIR sensors of the nodes displayed sporadic and erratic behavior when the ambient temperature was extremely low (in the region of $-5{ }^{\circ} \mathrm{C}$ ). In such cases, no movement was detected by the sensors, however, the radio transmission would still work. This condition was not consistent and occurred unpredictably. Sometimes, the PIR sensors would return to their normal operation when kept at room temperature. In some other cases the nodes themselves stopped functioning and did not respond to hardware resets. Our investigations also showed that the culprit was memory corruption, which may also be indirectly linked to cold weather conditions.

With respect to the performance of the system in terms of accurately detecting the number of cars there are certain limitations that need to be addressed in future. For example, when two cars are passing through the entry/exit point at the same time (i.e., one car is exiting the parking lot while one is entering at the same time) some false detections are observed. A possible solution to this problem is to use a pair of nodes on each edge of the roadway and dedicate each pair for detections in each direction (i.e., each pair of nodes will only detect movement in the lane that is closer to them). The nodes then need to negotiate with the nodes on the other side of the road to identify and resolve these detections.

\section{CONCLUDING REMARKS}

We have developed a WSN-based gate monitoring system and studied its application to monitoring of parking lots. Our system has been designed to be energy efficient, robust, and cost efficient. The design of the system incorporates features that allow it to be easily configured and parameterized for wide range of monitoring applications.

The results from our parking lot deployment suggests that there are significant patterns that emerge from the occupancy data, that can facilitate planning for future expansions of the parking lots and traffic management on special events. In future work, we plan to deploy a larger scale version of our system to monitor all the parking lots at our university. Other applications of our system includes monitoring the occupancy of public buildings and shopping malls.

\section{ACKNOWLEDGMENT}

We would like to thank University at Buffalo, Parking and Transportation Services for their help during our experiments.

\section{REFERENCES}

[1] R. Szewczyk, J. Polastre, A. Mainwaring, and D. Culler, Lessons from a Sensor Network Expedition, Feb 2004, vol. 2920/2004, pp. 307-322.

[2] A. Mainwaring, D. Culler, J. Polastre, R. Szewczyk, and J. Anderson, "Wireless sensor networks for habitat monitoring," 2002, pp. 88-97.

[3] B. T. Burrell, J. and R. Beckwith, Vineyard Computing: Sensor Networks in Agricultural Production, Jan-Mar 2004, vol. 3, pp. 38-45.

[4] A. Arora, P. Dutta, S. Bapat, and et.al., "A line in the sand: a wireless sensor network for target detection, classification and tracking," The International Journal of Computer and Telecommunications Networking, vol. 46, pp. 605-634, Dec 2004.

[5] A. A., E. E., and et. al., ExScal: elements of an extreme scale wireless sensor network, 2005, pp. 102-108.

[6] Z. Pala and N. Inanc, "Smart parking applications using rfid technology," Sep 2007, pp. 1-3.

[7] M. Caruso and L. Withanawasam, "Vehicle detection and compass applications using AMR magnetic sensors," 1999, pp. 1-3.

[8] S. Liu, "Robust vehicle detection from parking lot images," no. ECE2005-06, Dec 2005.

[9] H. Moon, R. Chellappa, and A. Rosenfeld, "Performance analysis of a simple vehicle detection algorithm," vol. 20, 2002, pp. 1-13.

[10] M. Chiu, R. Depommier, and T. Spindler, "An embedded real-time vision system for 24-hour indoor/outdoor car-counting applications," vol. 3 , Aug 2004, pp. 338-341.

[11] H. Lee, D. Kim, D. Kim, and S. Y. Bang, "Real-time automatic vehicle management system using vehicle tracking and car plate number identification," vol. 2, July 2003, pp. 353-356.

[12] J. Chinrungrueng, U. Sunantachaikul, and S. Triamlumlerd, "Smart Parking: An Application of Optical Wireless Sensor Network," Applications and the Internet Workshops, IEEE/IPSJ International Symposium on (SAINTW'07), vol. 52, p. 66, 2007.

[13] S. S. Kulkarni and M. Arumugam, "Ss-tdma: A self-stabilizing mac for sensor networks," in Sensor Network Operations, IEEE Press., 2005.

[14] C. Busch, M. Magdon-Ismail, F. Sivrikaya, and B. Yener, "Contentionfree mac protocols for wireless sensor networks." in DISC, 2004, pp. 245-259.

[15] CrossBow Techno., "http://www.xbow.com/products/product_pdf_files/ wireless_pdf/msp410ca_datasheet.pdf."

[16] P. Dutta, "On random event detection with wireless sensor networks," 2004.

[17] S. Bibyk, "www.ece.osu.edu/ bibyk/ee582/xscalemote.pdf."

[18] P. Dutta, M. Grimmer, A. Arora, S. Bibyk, and D. Culler, "Design of a wireless sensor network platform for detecting rare, random, and ephemeral events," vol. 3, Apr 2005, pp. 497-502. 